\title{
In situ STEM Investigation of Shape-Controlled Synthesis of Au-Pd Core- Shell Nanocubes
}

\author{
Nabraj Bhattarai and Tanya Prozorov
}

Emergent Atomic and Magnetic Structures, Division of Materials Sciences and Engineering, Ames Laboratory, Ames, IA 50011, USA.

Properties and applications of nanoparticles (NPs) strongly depend on their shape, size and crystalline structure. The fabrication of NPs with well-controlled shape, size and crystallinity with sufficient yield represents one of the main challenges of the nanoscience and nanotechnology. Despite the vast research, the nucleation and shape-selective growth mechanism of NPs from solution are not fully understood. The in situ scanning transmission electron microscopy (STEM) analysis has become one of the most important technique allowing dynamic investigation of NP growth.[1-3] We studied shape- and sizecontrolled in situ formation of Au-Pd core-shell nanocube from gold NPs.

The in situ STEM study was carried out by using a Continuous Flow Fluid Cell holder platform with the reagents sealed between the two $50 \mathrm{~nm}$-thick electron-transparent silicon nitride ( $\mathrm{SiN})$ membranes. Visualization of controlled shape-selective synthesis of Au-Pd core-shell nanocubes was performed with the FEI Tecnai $\mathrm{G}^{2}$ F20 STEM operated at $200 \mathrm{kV}$, using the high angle annular dark field (HAADF) STEM imaging mode. The ex situ STEM micrograph of core-shell nanocube (Figure 1), shows $\sim 20 \mathrm{~nm}$ cubes with uniform shapes and sizes. These nanocubes are formed from Au octahedral NPs with fast growth of $\mathrm{Pd}$ along $\langle 111\rangle$ directions than along $\langle 100\rangle$ direction, when Pd is added onto it and reduced by ascorbic acid (AA).[4] The liquid cell reaction visualized in the static mode is presented in Figure 2a, b. In this case, Au NPs were mixed with Pd precursor and AA and deposited in the SiN window and hermetically sealed. The liquid cell holder platform was inserted into the microscope and STEM analysis was carried out. The interaction of electron beam with the sample and the effect of electron dose are currently investigated. As seen in Figure 2a, b, while some core-shell NPs are formed with Au as core and Pd as the shell, the Pd layer is not uniform. Initially there were very few particles but after an hour several NPs were seen. More than $60 \%$ of NPs consisted predominantly of Pd (represented by black circles) indicating excess of $\mathrm{Pd}$ in the system. On the other hand, in the areas not directly exposed to the beam, well defined shape and sized NPs are present. In another static in situ experiment, the Pd concentration was reduced, yielding the core-shell structure shown in an inset of Figure 2a. The shape and size of these nanocubes are in good agreement with those obtained $e x$ situ. In order to elucidate the reaction mechanism of core-shell nanocube formation from Au NPs and visualize the reaction in real time, further experiments will involve controlled delivery of the reactants in situ.

\section{References:}

[1] K. L. Jungjohann et al, Nano Letters, 2013, 13, 2964-70.

[2] H.-G. Liao et al, Science, 2012, 336, 1011.

[3] T.J. Woehl et al, Scientific Report, 2014, 4, 6854.

[4] N. Bhattarai et al, Journal of Nanoparticle Research, 2013, 15, 1660.

[5] T.P. acknowledges support from the Department of Energy Office of Science Early Career Research Award, Biomolecular Materials Program. This work was supported by the U.S. Department of Energy, Office of Basic Energy Science, Division of Materials Sciences and Engineering. The research was performed at the Ames Laboratory, which is operated for the U.S. Department of Energy by Iowa State University under Contract No. DE-AC02-07CH11358. 


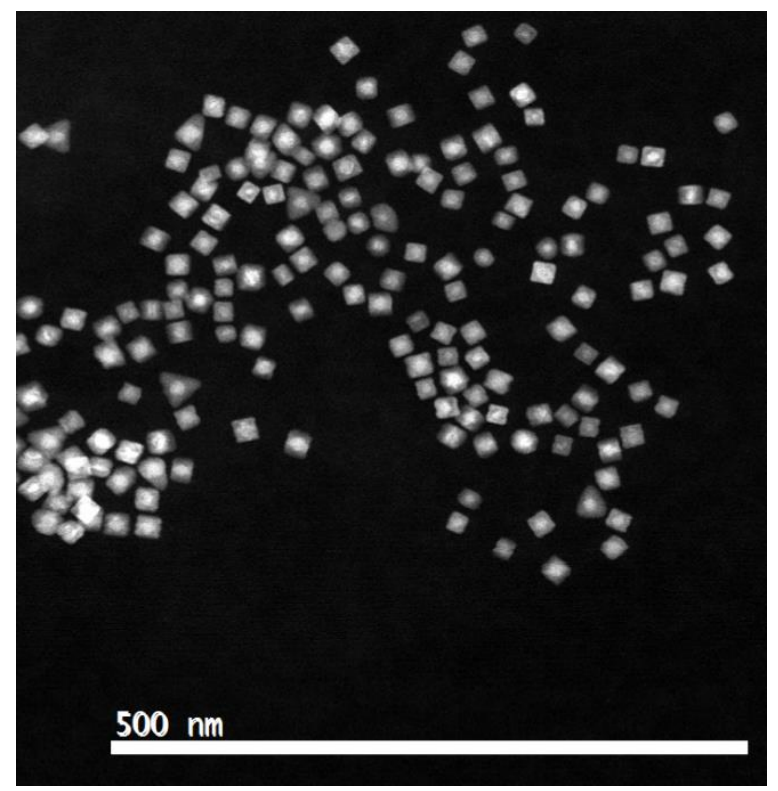

Figure 1: Ex situ HAADF STEM micrograph of Au-Pd core-shell nanocube as seen in S/TEM grid. The Z-contrast imaging showed that the nanocube is $\sim 20 \mathrm{~nm}$ in size with the core made from Au (higher atomic number) and the shell made from $\mathrm{Pd}$ (lower atomic number).
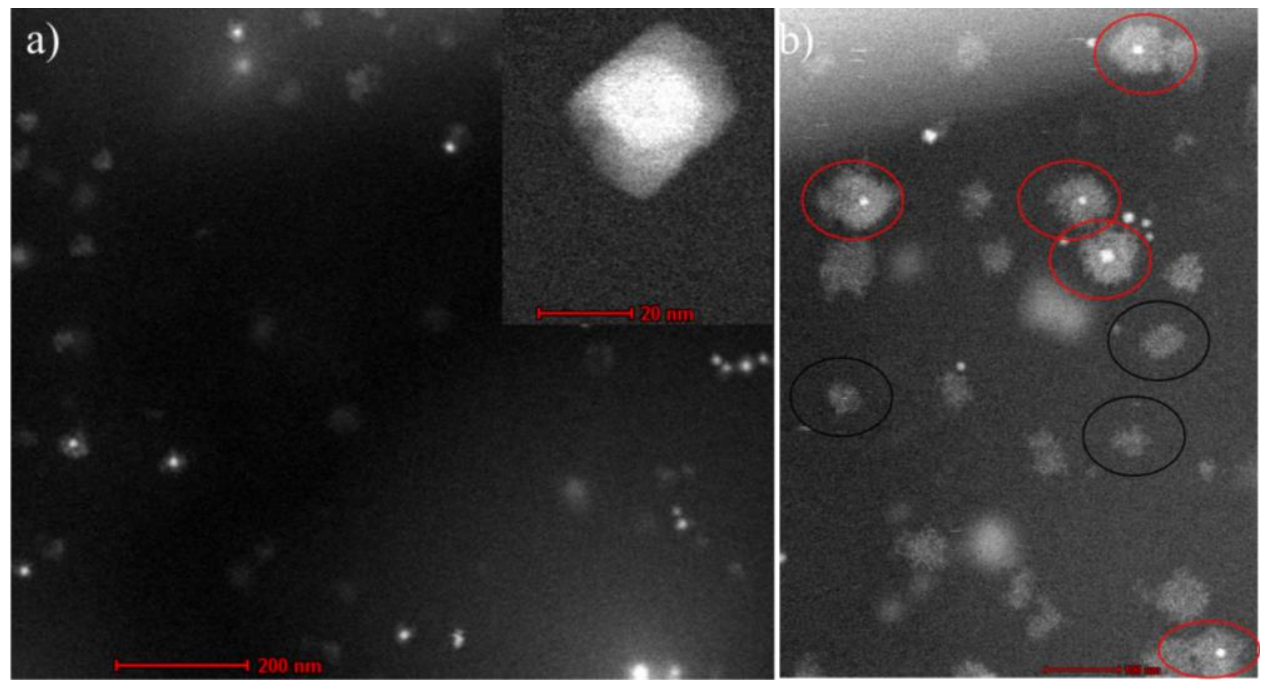

Figure 2: Low magnification in-situ STEM imaging of the formation of Au-Pd core-shell nanostructures from octahedral Au NPs. (a) Low magnification image shows that core-shell nanostructures are obtained with $\mathrm{Au}$ inside (core) and Pd outside (shell). A random area in Figure (a) is zoomed in and is presented in Figure (b) where the core-shell structure is clearly seen (represented by red circled regions). The coreshell ex-situ nanocubes are relatively smaller in size $\sim 20 \mathrm{~nm}$, while the in-situ (red circled) are larger in size $\sim 60 \mathrm{~nm}$. Some of the Pd nanocrystals were seen (represented by black black circles). Size controlled AuPd core-shell nanocube obtained from another static in situ experiment by changing the concentration of $\mathrm{Pd}$ precursor is inset in Fig. 2a, which shows the nanocube is made from Au core and some finite layer of Pd shell, indicating that the in situ experiment is close agreement with the ex situ experiments. 